\title{
Impactos da pandemia da Covid-19 na saúde das mulheres lésbicas
}

\author{
Letícia de Sousa Milanez ${ }^{1}$ \\ Breno de Oliveira Ferreira ${ }^{2}$ \\ José Ivo dos Santos Pedrosa ${ }^{3}$
}

Resumo: A pandemia da Covid-19 não afeta a todos da mesma maneira, o impacto criado pelas medidas sanitárias de isolamento físico adotadas no contexto atual de crise sanitária e política é mais marcante e cruel para as populações submetidas a processos anteriores de vulnerabilização e violência, como a das mulheres lésbicas, levando em consideração o cenário machista, racista e lesbofóbico em que vivemos. Este artigo tem por objetivo problematizar os impactos biopsicossociais da pandemia da Covid-19 na saúde das mulheres lésbicas, considerando a intersecção de diversos fatores que pode agravar ou atenuar estes impactos.

Palavras-chave: Mulheres Lésbicas; Covid-19; Pandemia.

\section{INTRODUÇÃO}

Em 11 de março de 2020, a Covid-19 foi caracterizada pela Organização Mundial de Saúde (OMS) como uma pandemia. O mundo inteiro entrou em alerta diante de uma doença pouco conhecida e que se apresentou de forma tão diferente nas pessoas. As medidas sanitárias adotadas pelas autoridades de saúde acarretaram várias transformações nas práticas e rotinas das comunidades e instituições públicas e privadas. No Brasil, fatores como a falta de informação, a propagações de fake news e o descaso do atual governo fazem com que a crise sanitária causada pela doença permaneça instaurada no país, que até o momento está em terceiro lugar com o maior número de casos da Covid-19 e com mais de 140.000 mortes, sem contar os impactos psicossociais, econômicos e de saúde agravados pelo atual cenário pandêmico.

A Covid-19 trouxe para população pânico, incerteza e insegurança, uma vez que, não é fácil combater uma doença, na qual não se tem ainda informações concretas sobre

\footnotetext{
${ }^{1}$ Mestranda em Ciências e Saúde pela Universidade Federal do Piauí - UFPI.

2 Professor-pesquisador efetivo da Universidade Federal do Amazonas (UFAM).

3 Professor Titular em Saúde Coletiva da Universidade Federal do Piauí (UFPI).

Vol. 03, N. 11, Jul. - Set., 2020 - http://periodicoscientificos.ufmt.br/ojs/index.php/rebeh/index
} 
o risco clínico, como também não se conhece com exatidão o padrão de transmissibilidade, infectividade, letalidade e mortalidade e até o momento não há vacinas ou medicamentos específicos disponíveis contra a doença (LIMA et al., 2020). O estudo de Carvalho et al. (2020) traz relatos de pânico e aflições das pessoas ao andar em locais públicos, uma vez que o vírus é percebido como "elemento intangível de um mal que circula por todos os lugares".

A grande propagação de informações nas redes sociais de tratamentos, formas de prevenção e transmissão da Covid-19, que vão de encontro às constatações científicas contribuíram no agravamento da crise sanitária e o aumento do número de mortes. As falas do atual presidente do Brasil, como "É só uma gripezinha", "Tenho histórico de atleta", diminuindo a dimensão de letalidade e mortalidade da doença e "E daí?", quando o questionaram sobre o rápido aumento de caso de óbitos, além do descredito na necessidade de isolamento físico como medida sanitária para o combate da transmissão do vírus SARS-CoV-2, mostra a marcante necropolítica promovida pelo governo (SANTOS; OLIVEIRA; OLIVEIRA-CARDOSO, 2020; THE LANCET, 2020).

Se por um lado, a grande disseminação de informações sobre um vírus "invisível" ainda pouco conhecido acarretou medo e pânico nas pessoas, por outro observa-se o descaso do governo diante de uma pandemia que traz consigo impactos negativos na vida das pessoas. A crise sanitária causada pelo vírus SARS-CoV-2 não atinge de forma igual à vida das pessoas. Nas populações que antes já viviam situações de vulnerabilidade e violência, e aqui citamos as populações LGBT+ (Lésbicas, Gays, Bissexuais, Travestis e Transexuais), estas situações são agravadas pelo cenário pandêmico e pela crise econômica e políticas já instaladas no Brasil desde o golpe político-institucional de 2016, que resultou no impeachment da presidenta Dilma Roussef. Atualmente o país está sob o comando da extrema-direita que busca constantemente a destruição das instituições democráticas (SANTOS; OLIVEIRA; OLIVEIRA-CARDOSO, 2020).

Em tempos de pandemia é desafiador promover saúde de forma universal, integral e equânime. E partindo do pressuposto que populações que já sofriam processos de vulnerabilização tendem a sofrerem mais com as consequências da crise sanitária, é de suma importância que fontes de desigualdades, como identidade de gênero e 
orientação sexual que escapam o binarismo sexual, sejam consideradas nas políticas públicas de saúde para minimizar os impactos da pandemia para os mais vulneráveis (SIGNORELLI et al., 2020).

No Brasil, em 2004, foi lançada a Política Nacional de Atenção Integral à Saúde da Mulher com objetivo de atender as mulheres em todas as suas especificidades rompendo com as fronteiras da saúde sexual e reprodutiva. Dentre estas especificidades está à garantia do atendimento à mulher lésbica de forma não discriminatória (BRASIL, 2004). Já em 2011, através da portaria nº 2.836/2011, o Ministério da Saúde lançou a Política Nacional de Saúde Integral de Lésbicas, Gays, Bissexuais, Travestis e Transexuais, como objetivo de estabelecer diretrizes e ações para as três esferas de governo no que se refere à promoção, prevenção e recuperação no cuidado em saúde, enfatizando a importância da redução das iniquidades decorrentes de identidade de gênero e orientação sexual (BRASIL, 2013). Estas políticas representam uma grande conquista na consolidação do direito à saúde das mulheres lésbicas, todavia é necessário que as diretrizes e as ações propostas sejam materializadas de acordo com a especificidade de cada mulher e com o contexto atual de crise sanitária que estamos vivenciando.

Segundo o estudo realizado pelo Coletivo \#VoteLGBT4 sobre os impactos da pandemia nas populações LGBT+, realizada entre os dias 28 de abril e 15 de maio de 2020 nas 5 regiões do Brasil, os três maiores impactos causados pela pandemia foram, piora na saúde mental, afastamento da rede de apoio e falta de fonte de renda. A pesquisa constatou que dos 9.521 entrevistados, $21,6 \%$ se encontram desempregados. Em relação às mulheres lésbicas $40 \%$ das entrevistadas declararam que possui uma renda menor ou igual a 01 salário mínimo, é sabido que grande parte destas mulheres não dispõe de trabalho formal e com a pandemia a perda de renda foi imediata, trazendo impactos na sua capacidade de sobrevivência e bem-estar. Outro determinante de saúde analisado foi à saúde mental, 42,72\% dos entrevistados relataram o aumento de

\footnotetext{
${ }^{4}$ \#VoteLGBT é uma coletivo que desde 2014 busca aumentar a representatividade de LGBT+ em todos os espaços, principalmente na política e tem por objetivo a geração cidadã de dados. Em $2016 \mathrm{com}$ a ajuda de mais de 60 pessoas voluntárias e a sob a orientação de professores do GPoPai-USP, foi criado um survey e aplicado na $20^{\mathrm{a}}$ Parada do Orgulho LGBT+ de São Paulo e na $14^{\mathrm{a}}$ Caminhada de Mulheres Lésbicas e Bissexuais, como objetivo inicial de descobrir quem frequentava as manifestações do orgulho LGBT+ e qual o seu perfil político. Hoje, o coletivo \#VoteLGBT busca levantar informações do contexto social/político vigente.
}

Vol. 03, N. 11, Jul. - Set., 2020 - http://periodicoscientificos.ufmt.br/ojs/index.php/rebeh/index 


\section{슈드태 \\ REVISTA BRASILEIRA DE ESTUDOS DA HOMOCULTURA}

problemas ligados à saúde mental e 54\% afirmaram precisar de apoio psicológico, além de relatar a dificuldade em acessar serviços de saúde especializados na área (\#VOTELGBT, 2020).

Em 1947 a OMS definiu saúde como "um estado de completo bem-estar físico, mental e social e não apenas a ausência de doença ou enfermidade" (OMS, 1978, p. 1). Pensar saúde em tempos de pandemia da Covid-19 é pensar não só em estar ou não infectado pelo vírus SARS-CoV-2, que pode acarretar inúmeros problemas de saúde de ordem biológica, como também é refletir sobre o impacto da doença frente ao agravamento de vários problemas de ordem social, econômica, mental, espiritual e política. É sabido que a pandemia da Covid-19 não afeta a todos da mesma maneira, o impacto criado pelas medidas sanitárias de isolamento físico adotadas no contexto atual de crise sanitária e política é mais marcante e cruel para as populações submetidas a processos anteriores de vulnerabilização e violência, como a das mulheres lésbicas, levando em consideração o cenário machista, racista e lesbofóbico em que vivemos. Diante do conceito de saúde proposto pela OMG e do cenário atual do Brasil, como podemos garantir saúde para as populações vulneráveis, como a das mulheres lésbicas no contexto pandêmico de crise sanitária, sabendo que estas mulheres há tempos sofrem com a invisibilidade no campo da saúde?

Nesta perspectiva, o presente estudo tem por objetivo problematizar os impactos biopsicossociais da pandemia da Covid-19 na saúde das mulheres lésbicas, considerando a intersecção de diversos fatores que pode agravar ou atenuar estes impactos. O artigo foi escrito, ora no tempo passado, ora no tempo presente, tendo em vista que até a conclusão deste, a pandemia da Covid-19 ainda estava em curso.

A interseccionalidade abordada no presente estudo visa dar instrumentalidade teórico-metodológica que nos auxilia a pensar como se entrecruzam e potencializam, os eixos de opressão, uma vez que, do ponto de vista analítico, permite identificação dos problemas sociais, capturando suas consequências estruturais e dinâmicas das complexas intersecções entre os vários eixos de subordinação (CRENSHAW, 2002). 
INTERSECCIONALIDADE E A INVISIBILIDADE DA SAÚDE DA MULHER LÉSBICA EM TEMPOS DE PANDEMIA

As mulheres lésbicas vivem em situação de vulnerabilidade, uma vez que permanecem em condição de marginalização imposta por uma sociedade heteronormativa (VALADÃO; GOMES, 2011). No campo da saúde, percebe-se que suas especificidades ainda não são observadas, fato que as tornam menos visíveis dentro do sistema, e assim, mais vulneráveis a fatores de risco quando comparadas a outras mulheres (CRISPIM et al., 2018).

A invisibilidade vivenciada pelas mulheres lésbicas é percebida e materializada no trabalho realizado por Facchini e Barbosa (2006), Dossiê Saúde de Mulheres Lésbicas Promoção da Equidade e da Integralidade, onde os autores apontam:

\begin{abstract}
A escassa, ou quase inexistente, produção científica abordando a temática saúde e homossexualidade feminina no Brasil; a inexistência de políticas de saúde consistentes para o enfrentamento das dificuldades e necessidades desta população; o precário conhecimento sobre suas demandas e a ausência de tecnologias de cuidado à saúde adequadas, aliados à persistência de prénoções e preconceitos, convertem-se, no âmbito da saúde pública, por exemplo, no desperdício de recursos, no constrangimento produzido no atrito das relações no interior dos serviços de saúde, na assistência inadequada, e muito provavelmente num contingente de mulheres que não obtiveram seu diagnóstico, nem seu tratamento (FACCHINI; BARBOSA, 2006, p. 29).
\end{abstract}

É notório o processo de vulnerabilização e invisibilidade da mulher lésbica dentro do campo da saúde e com a crise sanitária causada pela Covid-19 houve um agravamento destas situações. Mesmo que qualquer pessoa possa ser contaminada pelo vírus SARS-CoV-2, a intersecção de outros marcadores como gênero, orientação sexual, raça, deficiência, classe, geração, região de moradia e território explicam por qual motivo determinados grupos estão mais expostos à doença; e/ou possuem acesso litado aos serviços essenciais de saúde (BRITO et al., 2020).

Ser mulher em uma sociedade patriarcal e cisheteronormativa, por si só, já traz uma carga de inferiorizarão, e quando a mulher possui uma orientação sexual destoante da norma heterossexual há um aumento exponencialmente dessa vulnerabilização, e isso se multiplica caso ela seja de uma raça ou etnia também desvalorizada e subjugada socialmente. Essa realidade impõe dificuldades para a afirmação e construção da 
identidade lésbica, sendo que um dos instrumentos mais fortes utilizados pela sociedade patriarcal e cisheteronormativa é a invisibilidade (CARVALHO et al., 2020).

Segundo Silva e Gomes (2019), as mulheres lésbicas sofrem inúmeros preconceitos nos espaços públicos e privados causando violências físicas e psicológicas, a violência simbólica também encontra-se presente nos serviços de saúde, uma vez que é comum, esses serviços não considerarem as demandas e as especificidades dessas mulheres, contribuindo para o apagamento de sua existência.

Desde a introdução de medidas de isolamento físico para impedir a propagação da Covid-19, observa-se inúmeros impactos causados pela pandemia. Entre os efeitos podemos citar a violência contra as mulheres. Segundo a Ouvidoria Nacional dos Direitos Humanos (ONDH), do Ministério da Mulher, da Família e dos Direitos Humanos (MMFDH), entre os dias $1^{\circ}$ e 25 de março, mês da mulher, houve crescimento de $18 \%$ das denúncias de violência doméstica (BRASIL, 2020).

As mulheres lésbicas estão expostas a diferentes tipos de abuso relacionado à lesbofobia, estes abusos são cometidos em sua maioria por sua família, pela comunidade e por religiões fundamentalistas (EBIN; PRICE, 2019). A violência de gênero é uma realidade brasileira marcada por um contexto estruturalmente machista e patriarcal. Os casos de violência e feminicídio contra mulheres lésbicas anteriores à pandemia no Brasil já eram absurdos. Segundo o Dossiê sobre lesbocídio no Brasil: de 2014 até 2017, entre os anos 2000 a 2017 foram registrados 180 homicídios de lésbicas. Os lesbocídios são hegemonicamente tentativas de extermínio, catalogadas como crimes de ódio e motivadas por preconceito. São ações que demonstram a incapacidade de alguns segmentos da população de aceitarem as lésbicas e as respeitarem como pessoas em igualdade de direitos e deveres constitucionais (PERES; SOARES; DIAS, 2018).

Segundo Signorelli et al. (2020), a pessoa que anteriormente sofreu experiências de rejeição familiar ou de expulsão têm consequências maiores durante a pandemia, como por exemplo a falta de alguém para cuidar dela em caso de doença ou sequelas. Por outro lado, aquela que vive com familiares que não aceitam sua orientação sexual, as medidas de isolamento físico podem culminar em episódios de violência doméstica.

As novas regras de convívio impedem que as mulheres lésbicas tenham acesso às redes de apoio afetivas, como coletivos, amigos e movimentos militantes. A internet 
tornou-se o espaço de acolhimento, apesar de que a falta de privacidade em casa e as tensões nos relacionamentos familiares, podem afastar as pessoas destes momentos de interação com a rede afetiva (CARVALHO et al., 2020).

Em relação ao trabalho é sabido que a discriminação no ambiente de trabalho, a informalidade e o subemprego estavam presentes na vida das mulheres lésbicas antes da pandemia. Segundo Irigaray e Freitas (2011), estas situações podem ser atenuadas por conta de outros traços psicográficos, tais como: maior poder aquisitivo, pele branca e conformidade com os padrões estéticos brasileiros. Partindo deste pressuposto podemos afirmar que as dificuldades econômicas decorrentes da quarentena afetam a população como um todo, mas é evidente que a intersecção como outros fatores como gênero, orientação sexual e raça faz como que estas dificuldades sejam experienciadas de forma diferente, ou seja, mulheres lésbicas, pretas e periféricas sofrem mais com os impactos econômicos causados pela crise sanitária. A pesquisa realizada pelo coletivo \#VoteLGBT durante a pandemia como 9.521 pessoas LGBT+, sendo que $21.42 \%$ eram mulheres lésbicas apontou que uma em cada cinco pessoas LGBT+ não possui nenhuma fonte de renda individual (\#VOTELGBT, 2020).

Com a pandemia da Covid-19, a oferta, o acesso e a utilização de serviços de saúde sofrem um impacto negativo. Acessar os serviços de saúde para resolução de outros problemas não relacionado à infecção pelo vírus SARS-CoV-2 ficou bem mais complicado. Para as mulheres lésbicas, a invisibilidade e a violência simbólica sofrida nos espaços de saúde é uma realidade vivenciada antes mesmo da pandemia (VALADÃO; GOMES， 2011; FERREIRA; PEDROSA; NASCIMENTO， 2018; SILVA; GOMES, 2019), a generalização da orientação sexual como heterossexual por parte de alguns profissionais de saúde pode afastar e inibir este público (CABRAL et al., 2017).

A pesquisa de Crispim et al. (2018), mostra que o cuidado direcionado às mulheres lésbicas na maioria das vezes causa grande desconforto aos profissionais de saúde. Esta situação pode estar associada a fatos como: pensamento de que este padrão de sexualidade coloca à prova modelos hegemônicos de conjugalidade e família; heterossexualidade tida como único modelo de sexualidade "normal" aceito socialmente 
e despreparo por parte destes profissionais para lidar com mulheres lésbicas e bissexuais.

A invisibilidade e a violência simbólica vivenciada pelas mulheres lésbica são percebidas principalmente no atendimento ginecológico, quando estas se sentem violentadas com a realização de exames ginecológicos de forma dolorosa e inadequada ou quando a coleta de amostras citológicas para rastreamento do câncer do colo do útero não são realizadas, uma vez que se tem um imaginário comum de que essa população específica estaria menos susceptível a adquirir Infecções Sexualmente Transmissíveis (ISTs) (CABRAL et al., 2017; FERREIRA; PEDROSA; NASCIMENTO, 2018; RUFINO et al., 2018).

Diante disso, os profissionais de saúde devem ser capazes de conhecer e identificar as necessidades específicas sem criar barreiras. Faz-se necessário que as pessoas que estão envolvidas na atenção à saúde das mulheres lésbicas deixem de lado seus preconceitos e busquem conhecimentos através de atualizações, estudos e capacitações, que abordem à saúde destas mulheres.

Ressalto ainda que durante o período de enfrentamento da pandemia da Covid19 os estabelecimentos de saúde, e aqui damos ênfase à Atenção Básica, por ser a porta de entrada do Sistema Único de Saúde (SUS), permaneçam visibilizando a garantia de atendimento a outras doenças e demandas de saúde, como a atenção à saúde ginecológica e reprodutiva, prevenção e tratamento de doenças crônicas não transmissíveis, atenção à saúde mental e ao enfrentamento do abuso de álcool e outras drogas, além da prevenção e controle das violências.

\section{CONSIDERAÇÕES FINAIS}

Com intuito de minimizar as barreiras encontradas pelas mulheres lésbicas no atendimento nos serviços de saúde e agravadas pela pandemia da Covid-19 é necessário que algumas medidas sejam tomadas, a fim de garantir uma saúde integral, equânime e livre de preconceitos. Entres a ações efetivas para minimizar os impactos da pandemia podemos citar: a garantia da visibilidade das mulheres lésbicas, especialmente na coleta e monitoramento de dados epidemiológicos frente aos impactos da pandemia da Covid19, considerando a intersecção com outros fatores como gênero, raça, orientação sexual, Vol. 03, N. 11, Jul. - Set., 2020 - http://periodicoscientificos.ufmt.br/ojs/index.php/rebeh/index 
identidade de gênero, escolaridade e tanto outros marcadores sociais; promoção de abordagens de saúde sem discriminação ou estigmatização por parte dos profissionais de saúde que devem garantir um cuidado humanizado às mulheres lésbicas, abolindo a ênfase heteronormativa do atendimento ginecológico; garantir apoio psicossocial, com intuito de minimizar o sofrimento psíquico causado pelas medias de isolamento físico e garantir pacotes de proteção e cuidado social, como, por exemplo, a renda básica, principalmente para mulheres lésbicas mais vulneráveis.

\section{REFERÊNCIAS}

BRASIL, Ministério da Saúde. Secretaria de Atenção à Saúde. Departamento de Ações Programáticas Estratégicas. Política nacional de atenção integral à saúde da mulher: princípios e diretrizes. Brasília: Editora do Ministério da Saúde; 2004.

BRASIL. Coronavírus: sobe o número de ligações para canal de denúncia de violência doméstica na quarentena [Internet]. Brasil: Ouvidoria Nacional dos Direitos Humanos (ODNH), do Ministério da Mulher, da Família e dos Direitos Humanos (MMFDH), 2020. Disponível em: <https://www.gov.br/mdh/ptbr/assuntos/noticias/2020-2/marco/coronavirus-sobe-o-numero-de-ligacoes-para-canalde-denuncia-de-violencia-domestica-na-quarentena>. Acesso em: 25 set. 2020.

BRASIL. Ministério da Saúde. Política nacional de saúde integral de lésbicas, gays, bissexuais, travestis e transexuais. Brasília. $1^{a}$ Edição. $1^{a}$ Reimpressão. Ministério da Saúde, 2013.

BRITO, L. et al. "Impactos Sociais da Covid-19: uma perspectiva sensível às desigualdades de gênero". GT Bioética Abrasco, 2020. Disponível em: <https://www.arca.fiocruz.br/bitstream/icict/41375/2/ImpactosSociais.PDF>. Acesso em: 17 set. 2020 .

CABRAL, L. S. et al. "Homossexualidades femininas no contexto dos sistemas de informação de saúde". Rev enferm UFPE on line, Recife, v. 11, Sup.: 4, p. 1699-1707, abr., $2017 . \quad$ Disponível em: <https://periodicos.ufpe.br/revistas/revistaenfermagem/article/download/15267/18069> Acesso em: 17 set. 2020. https://doi.org/10.5205/reuol.10438-93070-1RV.1104sup201712.

CARVALHO, I. A. F. et al. "Invisibilidade lésbica e a interseccionalidade de opressões". 18 REDOR, p. 3008-3024, 2015. Disponível em: <http://www.ufpb.br/evento/index.php/18redor/18redor/paper/viewFile/2269/821>. Acesso em: 26 set. 2020.

CARVALHO, Mario et al. "METÁFORAS DE UM VÍRUS: REFLEXÕES SOBRE A SUBJETIVAÇÃO PANDÊMICA". Psicol. Soc., Belo Horizonte, v. 32, e020005, 2020. Disponível em: <http://www.scielo.br/scielo.php?script=sci_arttext\&pid=S0102- 
71822020000100404\&lng=es\&nrm=iso>. Acesso em: 21 set. 2020. http://dx.doi.org/10.1590/1807-0310/2020v32240308.

CRENSHAW, K. "Documento para o encontro de especialistas em aspectos da discriminação racial relativos ao gênero". Rev. Estud. Fem., Florianópolis, v. 10, n. 1, p. 171-188, Jan. 2002. Disponível em: <http://www.scielo.br/scielo.php?script=sci_arttext\&pid=S0104026X2002000100011\&lng=en\&nrm=iso>. Acesso em: 20 set. 2020. https://doi.org/10.1590/S0104-026X2002000100011.

CRISPIM, J. E. B. et al. "Assistência de enfermagem à mulher lésbica e bissexual na atenção básica: protocolo de atendimento". Revista de Pesquisa: Cuidado é Fundamental Online, [S.1.], v. 10, p. 34-39, jun, 2018. Disponível em: <http://www.seer.unirio.br/index.php/cuidadofundamental/article/view/7599>. Acesso em: 20 set. 2020. http://dx.doi.org/10.9789/2175-5361.2018.v10iEspecial.34-39.

CRISPIM, J. E. B., et al. "Assistência de enfermagem à mulher lésbica e bissexual na atenção básica: protocolo de atendimento". Revista de Pesquisa: Cuidado é Fundamental Online, [S.1.], v. 10, p. 34-39, june 2018. ISSN 2175-5361. Disponível em: $\quad$ http://www.seer.unirio.br/index.php/cuidadofundamental/article/view/7599>. Acesso em: 20 set. 2020. http://dx.doi.org/10.9789/2175-5361.2018.v10i3.34-39.

EBIN, C; PRICE, J. R. "The Great American Rights Bake Off: Freedom of Religion v. Freedom from Discrimination". New Political Science, v. 41, n. 4, p. 588-603, 2019. https://doi.org/10.1080/07393148.2019.1686736.

FACCHINI, R.; BARBOSA, R. M. Dossiê: Saúde das Mulheres Lésbicas promoção da equidade e da integralidade. Belo Horizonte: Rede Feminista de Saúde. 2006. Disponível

em: <http://www.cfess.org.br/arquivos/dossie_da_saude_da_mulher_lesbica.pdf >. Acesso em: 20 set. 2020 , p. 29.

FERREIRA, B. O.; PEDROSA, J. I. S.; NASCIMENTO, E. F. "Diversidade de Gênero e Acesso ao Sistema Único de Saúde". Rev. Bras. Promoç. Saúde [Internet], Fortaleza, v. 31, n. 1, p. 1-10, ago. 2017. Disponível em: <http://periodicos.unifor.br/RBPS/article/view/6726/pdf.> Acesso em: 23 set. 2020. https://doi.org/10.5020/18061230.2018.6726.

IRIGARAY, H. A. R.; FREITAS, M. E. "Sexualidade e organizações: estudo sobre lésbicas no ambiente de trabalho". Organ. Soc., Salvador, v. 18, n. 59, p. 625-641, Dez. 2011.

Disponível

em: <http://www.scielo.br/scielo.php?script=sci_arttext\&pid=S198492302011000400004\&lng=en\&nrm=iso>. Acesso em: 26 set. 2020. http://dx.doi.org/10.1590/S1984-92302011000400004.

LIMA, C. K. T. et al. "The emotional impact of Coronavirus 2019- nCoV (new Coronavirus disease)". Psychiatry Research, Elsevier Ireland, v. 287, n. 1, p. 1-2, 2020. <https://www.sciencedirect.com/science/article/abs/pii/S0165178120305163>. Acesso em: 20 set. 2020. https://doi.org/10.1016/j.psychres.2020.112915. 
ORGANIZAÇÃO MUNDIAL DE SAÚDE (OMS). Declaração de Alma-Ata: primeira conferência internacional sobre cuidados primários de saúde. Genebra, 1978, p.1.

PERES, M. C. C.; SOARES, S. F.; DIAS, M. C. Dossiê sobre lesbocídio no Brasil: de 2014 até 2017. Rio de Janeiro: Livros Ilimitados, 2018. 116 p.

RUFINO A. C. et al. "Disclosure of sexual orientation among women who have sex with women during gynecological care: a qualitative study in Brazil". J Sex Med, v. 15, n. 7, p. 966-973, 2018. Disponível em: <https://www.jsm.jsexmed.org/article/S17436095(18)30977-9/fulltext>. Acesso em: 17 set. 2020.

SANTOS, M. A.; OLIVEIRA, W. A.; OLIVEIRA-CARDOSO, E. A. "INCONFIDÊNCIAS DE ABRIL: IMPACTO DO ISOLAMENTO SOCIAL NA COMUNIDADE TRANS EM TEMPOS DE PANDEMIA DE COVID-19”. Psicol.

Soc., Belo Horizonte, v. 32, e020018, 2020. Disponível em $<$ http://www.scielo.br/scielo.php?script=sci_arttext\&pid=S0102-

71822020000100417\&lng=en\&nrm=iso>. Acesso em: 27 set. 2020. https://doi.org/10.1590/1807-0310/2020v32240339.

SIGNORELLI, M. et al. "The health of LGBTI+ people and the COVID-19 pandemic: A call for visibility and health responses in Latin America”. Sexualities, july, 2020. Disponível em: <https://journals.sagepub.com/doi/10.1177/1363460720942016> Acesso em: 17 set. 2020. https://doi.org/10.1177/1363460720942016.

SILVA, A. N.; GOMES, R. "Acesso de Mulheres lésbicas aos serviços de saúde à luz da literatura". Cien Saude Colet [periódico na internet], dez, 2019. Disponível em: $<$ http://www.cienciaesaudecoletiva.com.br/artigos/acesso-de-mulheres-lesbicas-aosservicos-de-saude-a-luz-da-literatura/17473?id=17473> Acesso em: 25 set. 2020.

THE LANCET. COVID-19 no Brasil: "E daí?". Lancet, Inglaterra, v. 395, n. 10235, p. 1461. Disponível em: <https://www.ncbi.nlm.nih.gov/pmc/articles/PMC7251993/>. Acesso em: 25 set. 2020. https://doi.org/10.1016/S0140-6736(20)31095-3.

VALADAO, R. C.; GOMES, R. "A homossexualidade feminina no campo da saúde: da invisibilidade à violência". Physis, Rio de Janeiro, v. 21, n. 4, p. 1451-1467, dez. 2011. Disponível em: <http://www.scielo.br/scielo.php?script=sci_arttext\&pid=S0103$73312011000400015 \& \operatorname{lng}=$ en\&nrm $=$ iso $>$.

Acesso em: 23 set. 2020. https://doi.org/10.1590/S0103-73312011000400015.

\#VoteLGBT. Diagnóstico LGBT+ na Pandemia: Desafios da comunidade LGBT+ no contexto de isolamento social em enfrentamento à pandemia de Coronavírus. Pesquisa Junho 2020. Disponível em: https://www.votelgbt.org/pesquisas Acesso em: 25 set. 2020. 


\begin{abstract}
The Covid-19 pandemic does not affect everyone in the same way, the impact created by the sanitary measures of physical isolation adopted in the current context of health and political crisis is more striking and cruel for the populations subjected to previous processes of vulnerability and violence, as that of lesbian women, taking into account the macho, racist and lesbophobic scenario in which we live. This article aims to discuss the biopsychosocial impacts of the Covid-19 pandemic on the health of lesbian women, considering the intersection of several factors that can aggravate or mitigate these impacts.
\end{abstract}

Keywords: Lesbian women; Covid-19; Pandemic.

Recebido: 07/10/2020

Aceito: 14/12/2020 